\title{
The influence of high addition soft multifocal contact lenses on visual performance
}

\author{
Sylwia Kropacz-Sobkowiak ${ }^{1,2,3}$, Anna Przekoracka-Krawczyk ${ }^{1,2}$, Krzysztof Michalak ${ }^{1,2}$, Andrzej Michalski ${ }^{4}$, \\ Karolina Kujawa ${ }^{1}$, Jan Olszewski ${ }^{3}$ \\ 'Laboratory of Vision Science and Optometry, Faculty of Physics, Adam Mickiewicz University, Poznan, Poland \\ ${ }^{2}$ Laboratory of Vision and Neuroscience, NanoBioMedical Center, Adam Mickiewicz University, Poznan, Poland \\ ${ }^{3}$ Department of Bionics and Bioimpedance, Poznan University of Medical Sciences, Poznan, Poland \\ ${ }^{4}$ Department of Ophthalmology, Chair of Ophthalmology and Optometry, Poznan University of Medical Sciences, Poznan, Poland
}

\begin{abstract}
Aim of the study: The purpose of this study was to investigate the influence of multifocal contact lenses with medium and high additions (ADD: $+2.00 \mathrm{D}$ and $+4.00 \mathrm{D}$ ) on short-term visual functions in a young, healthy adult population. Additionally, the impact of central zone size ( $3 \mathrm{~mm}$ and $4.5 \mathrm{~mm}$ ) on visual functions was analyzed. Material and methods: Each subject $(N=24)$ was fitted with three different designs of soft contact lenses: (1) distance power plano with ADD +2.00 D (Relax, SwissLens) - ADD2, (2) distance power plano with ADD +4.00 D (Relax, SwissLens) - ADD4, (3) single vision spherical plano lenses (Orbis, SwissLens) - ADD0. In each pair of lenses, near and distance visual acuity (VA), the consensual response of accommodation (using the photorefraction method), accommodation facility and peripheral contrast sensitivity were measured. Results: Mean distance VA decreased with lenses with a $3 \mathrm{~mm}$ central zone size compared to a $4.5 \mathrm{~mm}$ central zone size $(p=0.019)$ and with ADD2 power compared to ADD0 $(p=0.004)$ in both central zone size groups. There was no statistically signifi-
\end{abstract}

cant effect of ADD power or central zone size on near VA. The size of the central zone influenced contrast sensitivity functions (the area under the log contrast sensitivity function was 5.8 vs. 6.7 , for $3 \mathrm{~mm}$ and $4.5 \mathrm{~mm}$ central zone size, respectively, $p<0.001)$. The mean lag of accommodation increased with ADD $(p=0.003)$. The slope of the linear function of accommodation was lower for ADD2 and ADD4 lenses ( $p=0.005)$ and this effect was observed for both central zone sizes.

Conclusions: Higher addition (ADD4) powers influenced shortterm visual functions in a similar way to that of medium (ADD2) addition powers in tested multifocal lens. The decrease of distant VA and increase in accommodative lag were observed with higher and medium addition powers. Because the size of the central distance zone negatively influenced some visual functions, this parameter should be considered individually in the fitting of soft multifocal contact lenses for myopia control.

KEY WORDS: soft multifocal contact lenses, myopia control, visual functions.

\section{INTRODUCTION}

Over the last decades, many clinical trials and studies have been performed in the field of myopia control (MC), focusing in particular on the use of optical devices for slowing down myopia progression [1]. Currently, while no MC intervention is effective for all patients [2], results from many studies have shown a significant MC effect with optical interventions, especially multifocal soft contact lenses (MFSCLs) and orthokeratology (OK) [3]. Contact lenses (MFSCLs soft and $\mathrm{OK}$ ) are included in myopia management guidelines [4] and therefore cannot be ignored by clinicians. Optical devices for $\mathrm{MC}$ are used in eye care practices with the hope of arresting myopia progression among the young population. Although there are not many products registered specifically for $\mathrm{MC}$, clinicians often use off-label contact lenses for MC in their daily practice [5]. Results of a global survey among practitioners show that MC contact lens fitting has been increasing over the last 8 years, and that almost half of MC contact lens fits globally are MFSCL central distance design lenses [6]. More than $50 \%$ of contact lenses fitted for MC were rigid contact lenses, predominantly of OK design [6]. Soft contact lenses used for MC might have different designs, such as progressive multifocal design with center distance, alternating bifocal design or many variations thereof [7]. The main goal in all constructions 
is to create myopic blur on the peripheral retina, while correcting foveal refraction error at the same time [7]. MFSCLs for $\mathrm{MC}$ have the potential of a huge commercial success around the world due to ease of fit, almost immediate comfort and a possibility to be fitted not as custom made devices (unlike OK lenses). According to data from clinical trials, MFSCLs can slow down myopia progression by $21 \%$ to $77 \%$ [2], with the mean MC effect usually reported as 50\% [8]. The differences in the MC effect between the mentioned studies might be due to different designs of both clinical trials and the type of lenses. In addition, most studies do not customize contact lens parameters (e.g. central zone diameter, contact lens diameter, base curve or the power of addition) to subjects' individual cornea size and geometry, pupil size or level of myopia.

While the mechanism of action by which optical agents achieve MC is not yet fully understood [9], some potential models have been proposed. The studies show that manipulation of peripheral retina refractive status might influence axial eye growth, which is slower when relative myopic defocus is created on the wider field of the retinal periphery [10]. According to this model, when using MFSCLs for MC, a small central distance zone and a high addition (ADD) in the peripheral part of the lenses are used to maximize the effect of MC. In most studies on MC, small or medium ADD (from $+1.50 \mathrm{D}$ to $+2.50 \mathrm{D}$ ) in MFSCLs with a fixed central distance zone size (usually from $3.0 \mathrm{~mm}$ to $3.5 \mathrm{~mm}$ ) was used [7]. The reason for using small and medium $\mathrm{ADD}$ is the belief that high ADD might disturb visual functions, such as near visual acuity (nVA)/distance visual acuity (dVA), contrast sensitivity, accommodation and near phoria. There is also a concern that patients might feel discomfort wearing high ADD lenses [11]. Therefore, practitioners might be reluctant to use high ADD MFSCLs for myopia control in daily practice. The purpose of this study was to investigate the influence of medium and high ADDs (+2.00 D and +4.00 D), and different central distance optical zone sizes $(3.0 \mathrm{~mm}$ and $4.5 \mathrm{~mm})$, in the MFSCLs dedicated for MC on short-term visual functions.

\section{MATERIAL AND METHODS}

\section{Subjects and inclusion criteria}

Non-presbyopic adult subjects among the student population of Adam Mickiewicz University of Poznan participated in the study. The inclusion criteria were: emmetropia, myopia not greater than $6.00 \mathrm{D}$, regular astigmatism not greater than $-1.00 \mathrm{D}$, the best-corrected dVA and nVA $0.0 \operatorname{logMAR}$ $(20 / 20)$ or better; no binocular vision or accommodative problems; no history of ocular or systemic diseases and no medications used that could contraindicate with contact lens wear and/or alter visual function measurements. Previous contact lens wearing experience was not required. Overall, 24 subjects from 28 were enrolled in this study and all of them completed the examination. Among them, 20 were female $(83.3 \%)$ and 4 were male $(16.6 \%)$, with a mean age of 23.9 (range from 20 to 36 ) years old. Sixteen subjects were emmetropic (emmetropia defined as refractive error between $+0.75 \mathrm{D}$ and $-0.50 \mathrm{D}$ ) and 8 subjects were myopic (refractive error lower than $-0.50 \mathrm{D})$. The mean subjective spherical refractive error was $-1.08 \pm 1.83 \mathrm{D}$, the mean cylinder $-0.25 \mathrm{D}$ and the mean dVA was $-0.17 \log M A R$.

Contact lens movement and centration observed in a slit lamp examination were within normal limits. The average pupil size in photopic light conditions measured by topography (Keratograph 4, OCULUS Optikgeräte GmbH, Germany) was $3.29 \pm 0.43 \mathrm{~mm}$. Ocular pathology was excluded by ophthalmological examination.

The study protocol followed the tenets of the Declaration of Helsinki and has been approved by Poznan University of Medical Science research ethics committee. All subjects received an explanation about the nature of the study and provided written consent.

\section{Contact lenses}

Custom made MFSCLs or single vision spherical contact lenses (SVS) from SwissLens (SwissLens SA, Prilly, Switzerland) were used. MFSCLs (with plano power for distance) were designed with two different central distance zone diameters: $3.0 \mathrm{~mm}$ and $4.5 \mathrm{~mm}$ and two different ADDs in the peripheral part of the lenses: $+2.00 \mathrm{D}$ and $+4.00 \mathrm{D}$ ADD, hereafter referred to as ADD2 and ADD4, respectively. SVS plano lenses (hereafter referred to as ADD0) were used as control lenses. Subjects were divided randomly into two groups using MFSCLs with different sizes of the central zone (CZ) - Group 1: $\mathrm{CZ}=3.0 \mathrm{~mm}$, Group 2: $\mathrm{CZ}=4.5 \mathrm{~mm}$. Visual parameters of all subjects were measured with MFSCLs with two ADDs (ADD2 and $\mathrm{ADD} 4$ ) and with a control lens (ADD0) in alternate order.

The design of MFSCLs was with a polynomial progression zone, in which the power progression is faster in higher additions. All parameters and design details of the lenses used are presented in Table I, and the construction is presented in the paper of Blaser and Sexton [12] and of Montani and Blaser [13].

\section{Study design}

This was a prospective randomized and double-blinded study. All subjects were appointed for 3 visits ( 1 enrollment visit and 2 measurement visits). All the measurements were performed in the same room with the same light conditions, by the same researcher and using the same methods and

Table I. Technical details and parameters of the lenses used in the study

\begin{tabular}{|l|c|c|}
\hline Parameter & SVS lenses & MF lenses \\
\hline Commercial name & Orbis & Relax \\
\hline Material & $\begin{array}{c}\text { Contaflex GM3 58\% } \\
\text { (Acofilcon A) }\end{array}$ & $\begin{array}{c}\text { Contaflex GM3 58\% } \\
\text { (Acofilcon A) }\end{array}$ \\
\hline Water content & $59 \%$ & $59 \%$ \\
\hline Base curve & $8.6 \mathrm{~mm}$ & $8.6 \mathrm{~mm}$ \\
\hline Diameter & $14.2 \mathrm{~mm}$ & $14.2 \mathrm{~mm}$ \\
\hline Distance power & Plano & Plano \\
\hline Distance zone diameter & N/A & $3.0 \mathrm{~mm}$ and $4.5 \mathrm{~mm}$ \\
\hline Near ADD power & $\mathrm{N} / \mathrm{A}$ & $+2.00 \mathrm{D}$ and $+4.00 \mathrm{D}$ \\
\hline
\end{tabular}


equipment. The subjects were coded by numbers and symbols and both experimenters and subjects had no information on what $\mathrm{ADD}$ was used during the measurements.

At the enrollment visit, all subjects underwent refractive assessment, accommodation and binocular function examination, corneal topography measurements and full evaluation of anterior and posterior eye segments. Contact lens movement and centration were evaluated in slit lamp examination. For the assessment of mutual location of the pupil and the MFSCL central zone, corneal topography with the lenses on the eye and pupil size measurements in photopic conditions using Keratograph 4 (OCULUS Optikgeräte GmbH, Germany) were performed. Measurements were taken after one hour of adaptation.

\section{Procedure}

Each subject underwent a series of visual tests (dVA, nVA, peripheral contrast sensitivity test (PCS), accommodative response (AR) and accommodative facility (AF)) fitted with lenses with each addition (ADD0, ADD2, and ADD4) and with spectacles, if needed for correction of refractive error. Binocular VA was measured at a distance of $5 \mathrm{~m}$ using a tumbling E chart displayed on a Frey CP-600P chart panel (dVA) and at $0.4 \mathrm{~m}$ using the LEA SYMBOLS Near Vision CardLea VA test (nVA) and the dVA/nVA results were converted to $\log$ MAR units. Both tests were performed in high contrast in photopic conditions.

The PCS test was designed based on the method used by Schumacher et al. [14].

The measurement was performed binocularly in a dark room after 5 minutes of adaptation (the average luminance level of the monitor was $90.16 \mathrm{~cd} / \mathrm{m}^{2}$ ). The monitor ( $19^{\prime \prime}$ iiyama HM903DT A CRT, resolution $1920 X 1440$ px, refresh rate $80 \mathrm{~Hz}$ ) was located $1.5 \mathrm{~m}$ away from the subject. The stimuli were created by vertical Gabor patches (size $0.7 \mathrm{deg}$ ) presented on a grey background in one of four positions (upper left, upper right, lower left or lower right). The Gabor patches were presented with 3 degrees eccentricity from the fixation cross ( 1 deg size). The spatial frequencies of the Gabor patches were $2.0,4.5,6.0,7.5$ and 10 cycles per degree (cpd). The Gabor patches were created with the online generator (http://www. cogsci.nl/gabor-generator) and the stimulus display was programmed and recorded in Presentation v1.9 software (Neurobehavioral Systems, Inc, Berkeley, CA, USA). The subject was asked to detect the Gabor patch by pressing one of the buttons on the numeric keyboard $(7,9,1,3)$. A new Gabor patch was displayed after the subject's response or after 1.5 seconds from the previous stimulus. The spatial frequency contrast threshold was determined using the staircase method.

Binocular AF was measured using text with 20/30 size letters presented at a distance of $0.4 \mathrm{~m}$ from the subject, and with a $\pm 2.00 \mathrm{D}$ spherical flipper. The position of the flipper was changed after the subject reported a clear image. The number of cycles (changes from $+2.00 \mathrm{D}$ to $-2.00 \mathrm{D}$ ) per minute was noted. This test was performed in a light room condition.
Consensual AR was measured using the photo-refraction method (PlusOptix A09, Plusoptix Inc. Atlanta, GA, USA). The refractive state of the left eye was measured in the subject's habitual correction, and the photo-refractor was aligned with the pupil center of that eye. The right eye of the subject was wearing the tested contact lens and looking at the target. The target consisted of two lines of a 4 letter matrix on a black background (each letter high was 0.36 degrees of visual angle), displayed on a notebook LCD screen (MacBook Pro 13", Apple Inc. Cupertino, CA, USA). The subject was instructed to keep the letters clear and to read them for the entire measurement time. A special separator was used to prevent the subject from looking at the target using the left eye. The refractive state of the left eye was measured with the lens off. The target for the accommodation was placed at $5 \mathrm{~m}, 1 \mathrm{~m}$, and $0.4 \mathrm{~m}$ distances from the subject. Three measurements were averaged for each distance. The distance of $5 \mathrm{~m}$ was treated as a reference refractive state, and the accommodative response was calculated as the difference between $5 \mathrm{~m}$ and $1 \mathrm{~m}$ ( $1 \mathrm{D}$ accommodative stimulus), and between $5 \mathrm{~m}$ and $0.4 \mathrm{~m}$ (2.5 $\mathrm{D}$ accommodative stimulus).

The subject's head was stabilized using a chin and forehead rest with a divider mask. The test was performed in a dark room.

\section{Statistical analysis}

Statistical analyses of data were performed using STATISTICA v.13.1 software (StatSoft). The Shapiro-Wilk test was used to assess the normality of data distribution. Depending on the results either the non-parametric Friedman test (for nVA) or ANOVA with repeated measurements (for other parameters) was used. Defined factors were: group (CZ3 and CZ4.5), ADD (ADD0, ADD2, ADD4) and stimulus for accommodation for the lag of accommodation parameter. The Tukey test was used in the post-hoc analyses. Differences were considered significant if the $p$-value was equal to or lower than 0.05 .

\section{RESULTS}

\section{Visual acuity}

Results of logMAR dVA (visual acuity) are displayed in Figure 1A. In general, mean dVA was worse for MFSCLs with CZ3 compared to CZ4.5 ( -0.12 vs. $-0.19 ; F(1,22)=6.38$, $\left.p=0.019, \eta^{2}=0.22\right)$. dVA was also affected by additions used in that $\mathrm{ADD} 2$ lenses decreased dVA compared to ADD0 lenses $(-0.17$ vs. -0.13 , post-hoc: $p=0.004)$, while there was no difference in dVA between ADD0 and ADD4 ( -0.17 vs. -0.16 , post-hoc: $p=0.481)$. This effect was found in both central zone sizes (CZ3 and CZ4.5), which was indicated by the insignificant group $\times \operatorname{ADD}$ interaction $\left(F(2,44)=0.42, p=0.663, \eta^{2}=0.02\right)$.

Results of logMAR nVA are presented in Figure 1B. Similarly as for dVA, ADD2 lenses slightly decreased nVA compared to ADD0 and ADD4 lenses for both groups (CZ3 and CZ4.5), but this effect failed to reach statistical significance (mean nVA for CZ3: $-0.05,-0.03,-0.05$ for ADD0, ADD2 and $\mathrm{ADD} 4$ respectively; $\chi^{2}=0.87, p=0.648$; mean $n V A$ for $\mathrm{CZ} 4.5$ : -0.07 for ADD0, ADD2 and ADD4, $\chi^{2}=2.00, p=0.368$ ). 


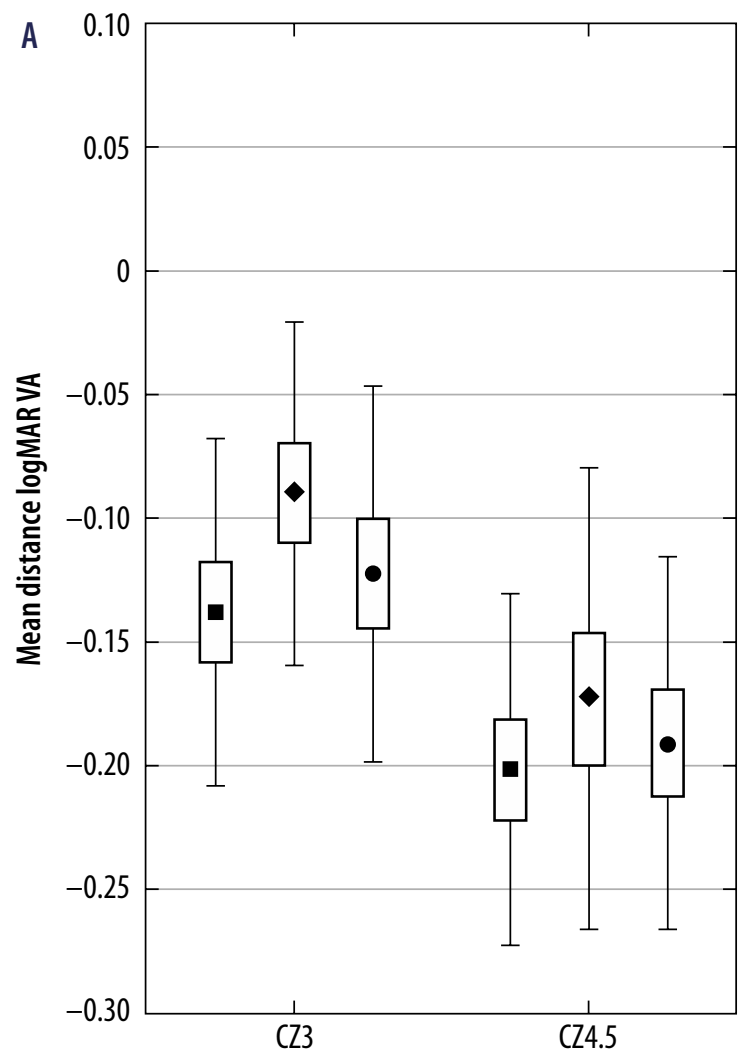

$\square$ ADD0 $\square$ ADD2 $\square$ ADD4

Type of contact lenses

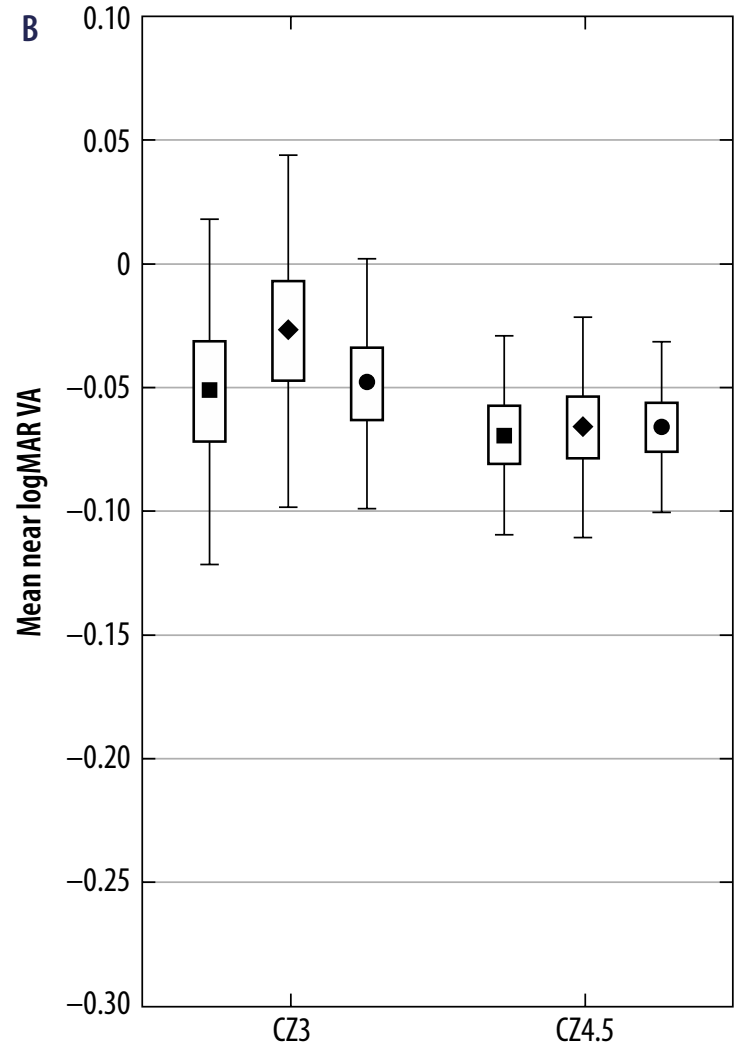

$\square$ ADD0 $\square$ ADD2 $\square$ ADD4

Type of contact lenses

Figure 1. Mean logMAR dVA (A) and nVA (B) in lenses with ADD0, ADD2 and ADD4 in groups with central zone size diameter 3.0 (CZ3) and 4.5 mm (CZ4.5). Error rectangles represent the standard deviation and whiskers represent standard error of the mean

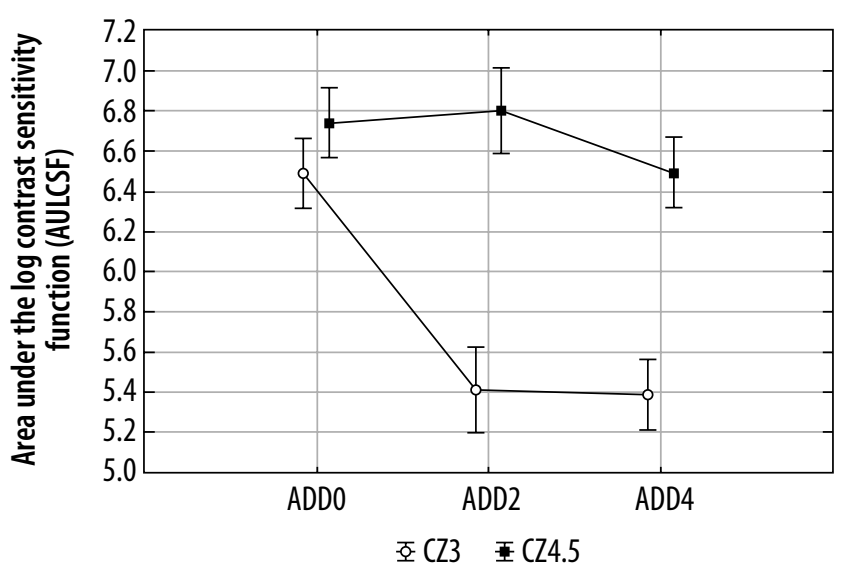

Figure 2. Area under the log contrast sensitivity function (AULCSF) for ADD0, $A D D 2$, and $A D D 4$ in groups $C Z 3$ and $C Z 4.5$. Whiskers represent the standard error of the mean

\section{Peripheral contrast sensitivity}

Peripheral contrast sensitivity (PCS) results are shown in Figure 2. Statistical analysis of the area under the log contrast sensitivity function (AULCSF) revealed significantly lower values for CZ3 compared to CZ4.5 (5.8 vs. 6.7 for CZ3 and CZ4.5 respectively, $\left.F(1,22)=17.18, p<0.001, \eta^{2}=0.44\right)$. AULCSF was reduced with increasing additional power $(6.6,6.1,5.9$ for $\mathrm{ADD} 0, \mathrm{ADD} 2$ and $\mathrm{ADD} 4$, respectively, $F(2,44)=14.46$,

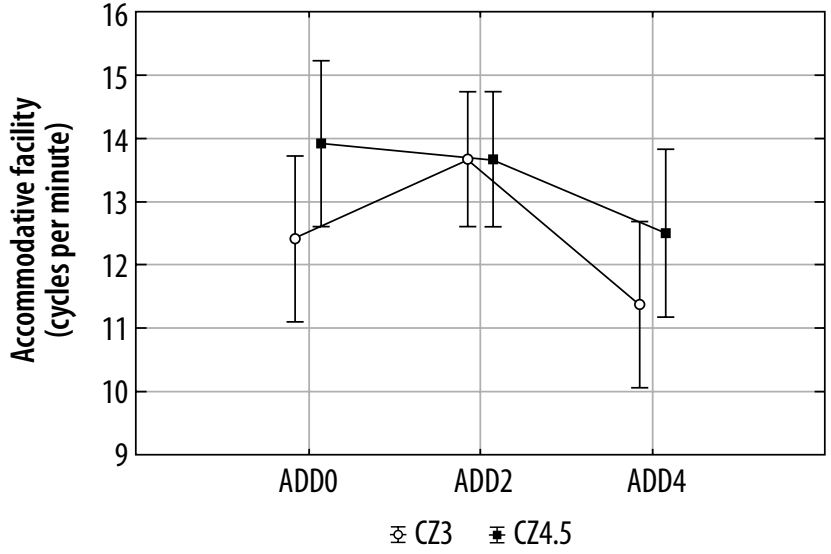

Figure 3. Accommodative facility (AF) for ADDO, ADD2, and ADD4 in groups $C Z 3$ and CZ4.5. Whiskers represent the standard error of the mean

$\left.p<0.001, \eta^{2}=0.40\right)$. Group $\times$ ADD interaction showed that AULCSF changed with three ADDs differently for CZ3 and CZ4.5 groups $\left(F(2,44)=10.0, p<0.001, \eta^{2}=0.32\right)$. Post-hoc analysis demonstrated that mean AULCSF for ADD0 was comparable between CZ3 and CZ4.5 groups (6.5 vs. 6.7 for $\mathrm{CZ3}$ and CZ4.5 respectively, $p=0.931)$. In the CZ3 group, both ADD2 and ADD4 lenses decreased AULCSF equally compared to ADD0 lenses (6.5, 5.4, 5.4 for ADD0, ADD2 and 
Table II. Mean lag of accommodation values for groups CZ3 and CZ4.5, different accommodative stimuli (1 D, $2.5 \mathrm{D})$ and different types of lenses (ADD0, ADD2 and $A D D$ 4). Values in bold represent statistically significant differences in the lag of accommodation for different types of lenses

\begin{tabular}{|l|l|l|}
\hline $\begin{array}{l}\text { Accommodative stimulus in different types } \\
\text { of lenses/groups }\end{array}$ & $\mathrm{CZ3}$ & $\mathrm{CZ4.5}$ \\
\hline 1D ADD0 & 0.59 & 0.64 \\
\hline 1D ADD2 & 0.74 & 0.86 \\
\hline 1D ADD4 & 0.85 & 0.80 \\
\hline 2.5 D in ADD0 & 0.77 & 0.52 \\
\hline 2.5 D in ADD2 & 1.12 & 0.95 \\
\hline 2.5 in ADD4 & 0.85 & 1.11 \\
\hline
\end{tabular}

ADD 4 respectively, post-hoc: $p<0.001)$. In contrast, in the CZ4.5 group, the slightly decreased AULCSF was observed only with ADD4, and this effect was not statistically significant (6.7, 6.8, 6.5 for $\mathrm{ADD} 0, \mathrm{ADD} 2$ and $\mathrm{ADD} 4$ respectively, post-hoc: $p>0.549)$.

\section{Accommodative facility}

Accommodative facility (AF) values for both central zone sizes and additions used are shown in Figure 3. Mean AF for CZ3 was lower than for CZ4.5 (12.5 vs. 13.4 cycles per minute for CZ3 and CZ4.5, respectively), but this difference was not statistically significant $\left(F(1,22)=0.63, p=0.435, \eta^{2}=0.03\right)$. $\mathrm{AF}$ was also not affected by additions used, which was proved by the not statistically significant main effect of $\operatorname{ADD}(F(2,44)$ $\left.=1.13, p=0.321, \eta^{2}=0.05\right)$ and the not statistically significant group $\times \operatorname{ADD}$ interaction $\left(F(2,44)=0.22, p=0.751, \eta^{2}=0.01\right)$.

\section{Accommodative response}

The accommodative lag and slope of a linear function of the accommodative response (AR) were analyzed, and the results are presented in the Figures. In addition, values for accommodative lag are shown in Table II.

Mean lag of accommodation (see Figure 4B and 4C) was nearly equal for both central zone sizes ( 0.82 vs. 0.81 for CZ3 and CZ4.5, respectively, $\left.F(1,22)<0.01, p=0.957, \eta^{2}=0.01\right)$. The ADD placed at the peripheral part of the contact lens increased the mean lag of accommodation $(0.63 \mathrm{D}, 0.90 \mathrm{D}, 0.92 \mathrm{D}$ for ADD0, ADD2 and ADD4, respectively, $F(2,44)=6.59$, $\left.p=0.003, \eta^{2}=0.23\right)$. Post-hoc analysis indicated a significant difference between ADD0 and ADD2 $(p=0.007)$ as well as between ADD0 and ADD4 $(p=0.010)$, but there was no significant difference between ADD2 and ADD4 $(p=0.987)$. Moreover, a significant interaction between group, ADD and stimulus for accommodation was found $\left(F(2,44)=4.70, p=0.014, \eta^{2}=0.18\right)$. As can be seen in Figure $4 \mathrm{C}$ and $4 \mathrm{D}$, the increase in lag of accommodation with ADDs was observed with both central zone sizes; however,

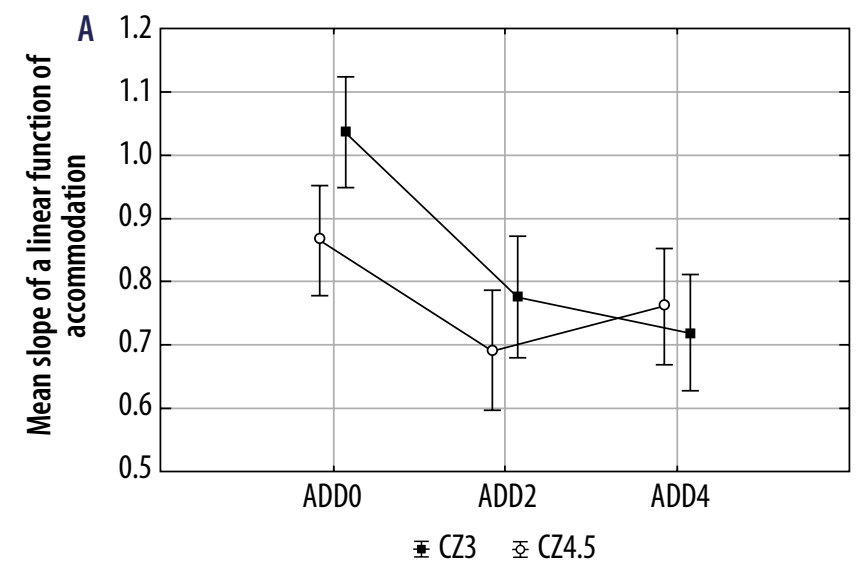

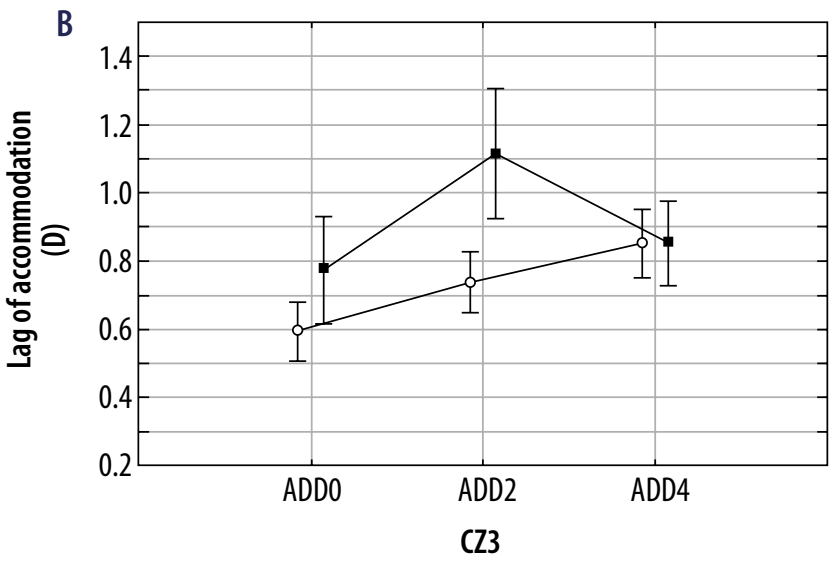

호 $1 \mathrm{D}$ 2.5D

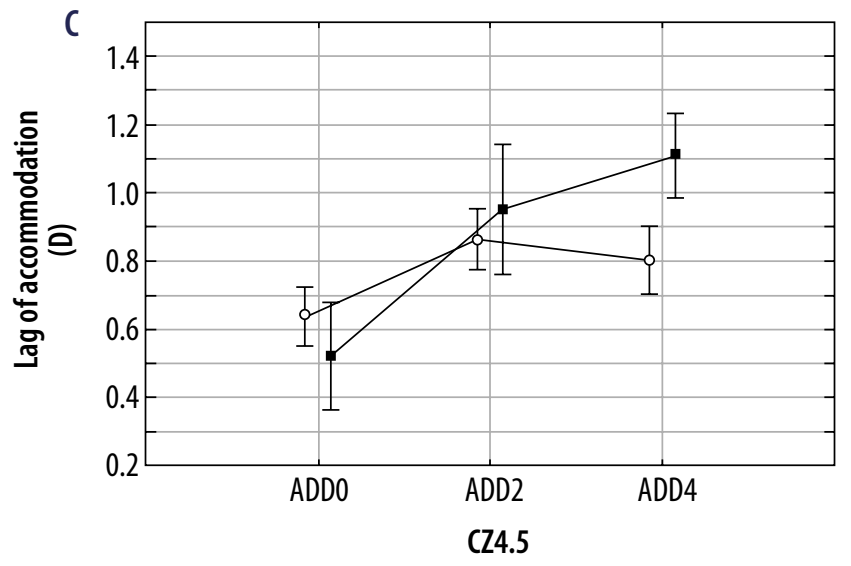

호 $1 D$ ㄴ.5 D

Figure 4. Mean slope of a linear function of accommodation $(A)$ and mean accommodative lag $(B, C)$ for lenses with ADD0, ADD2 and ADD4 in the groups with central zone size diameter 3.0 (CZ3) and $4.5 \mathrm{~mm}$ (CZ4.5). Whiskers represent the standard error of the mean 
post-hoc analysis revealed that this effect was significant only in the CZ4.5 group for 2.5 D stimulus of accommodation between ADD0 and ADD2 $(p=0.019)$ and between ADD0 and ADD4 $(p=0.004)$, but not between ADDs in CZ3 $(p>0.050)$. The mean slope of linear function of accommodative response was comparable for both central zone sizes (Figure $4 \mathrm{~A}, 0.77$ vs. 0.84 for CZ3 and CZ4.5, respectively, $\left.F(1,22)=0.51, p=0.500, \eta^{2}=0.02\right)$. However, this parameter was dependent on the ADD used. The slope of the function was lower for ADD2 and ADD4 (Figure 4A, 0.73 and 0.74 for $\mathrm{ADD} 2$ and ADD4, respectively) than for ADD0 (0.95), as was revealed by the significant main effect of $\operatorname{ADD}(F(2,44)=5.95$, $\left.p=0.005, \eta^{2}=0.21\right)$ and post-hoc test (ADD0 vs. ADD2: $p=0.011$ and ADD0 vs. ADD4: $p=0.014)$. The insignificant $\mathrm{ADD} \times$ group interaction showed that this effect occurred with both central zone sizes (CZ3 and CZ4.5, $F(2,44)=1.12$, $\left.p=0.336, \eta^{2}=0.05\right)$.

\section{DISCUSSION}

The purpose of this study was to assess the effect of MFSCLs dedicated to MC with medium (ADD2) and high (ADD4) addition powers on basic visual functions. An additional goal was to compare the influence of different central distance zone diameters on these functions. There are many MFSCL products designed and/or used for MC, which are accessible in some markets (Europe, North America, Australia). In many of these MC optical devices, a clinician can choose some of the construction parameters, such as the power of peripheral addition. According to the concept of peripheral myopic defocus having an inhibitory effect on axial eye growth [10], high addition MFSCLs could have a potentially stronger/ wider MC effect than lower ADDs used in standard MFSCLs, due to greater myopic defocus created on the peripheral retina [15]. Therefore, we decided to compare the influence of the frequently used power of peripheral ADD (ADD2) [1] with high power (ADD4) of peripheral addition. We found that the special construction of MFSCLs dedicated to MC (fast progress of peripheral addition) investigated in this study influenced high contrast dVA, but only with ADD2 and surprisingly not with ADD4 (Figure 1A). This effect was found with both central zone sizes (CZ3 and CZ4.5). Previous studies [11] on MFSCLs with slightly different designs revealed a decrease of low and high contrast dVA with $+1.50 \mathrm{D}$ and +3.00 D ADD MFCLs, and the dVA reduction was significant and sustained after two weeks of wear for lenses with +3.00 D ADD. In another study [16] the researchers found a significant decrease of dVA in MFSCLs and dual-focus lenses with +2.00 D ADD, only under low-illumination and low-contrast conditions. Other studies $[17,18]$ found no difference in high contrast dVA between +2.00 D ADD and VA in +2.50 D ADD lenses, but the researchers had to make over-refraction (from $-0.50 \mathrm{D}$ to $-0.75 \mathrm{D})$ to obtain good VA [17]. There is no consistency in the literature about the influence of MFSCLs used for MC on $\mathrm{dVA}$, probably due to the different designs of the lenses used and/or study protocols. Our main interest was to investigate the influence of high power of ADD on dVA. The results indi- cated that, since dVA was significantly worsened with ADD2 but not with ADD4 when compared to SVS (ADD0), a peripheral image blur created by the high ADD in the MFSCLs may be somehow "ignored" by the visual system, even more than a blurred image with the lower ADD (ADD2). Moreover, we observed that decreased dVA was present with MFSCLs with ADD2 regardless of the central zone size. There was also no influence of the lenses on the nVA regardless of the power of addition or central zone diameters used, which is in agreement with Przekoracka et al. [19].

Visual acuity testing is used in standard examinations, but the sensitivity of the test is not high, so the determination of the contrast sensitivity threshold seems to be a better method for detecting small changes in visual performance [11, $16,20]$. Since we used high ADD powers with fast peripheral progression, a decrease in peripheral contrast sensitivity was expected. We found that both additions used influenced PCS, but smaller central zone sizes ( $3 \mathrm{~mm}$ ) impaired this function more than bigger central zone size $(4.5 \mathrm{~mm})$, probably due to higher optical aberrations [20], which could be expected with MFSCLs [21]. Similar findings, but measuring contrast sensitivity in the central visual field, were reported by Gong et al. [22], who used MFSCLs with $+2.00 \mathrm{D}$ and $+2.50 \mathrm{D}$ ADDs compared to SVS control lenses. Other studies reported decreased low contrast VA in MFSCLs with $+3.00 \mathrm{D}$ $\mathrm{ADD}[20]$ and with +2.00 D ADD lenses used for MC [16]. The mentioned studies indicate that MFSCLs, even with medium ADDs, influence low contrast VA, but, interestingly, with high ADD, the reduction in low contrast VA was similar to lower ADD. This finding was similar to those reported by Przekoracka et al. [19], where peripheral contrast sensitivity was examined with high ADD MFCLs of the same design. However, they used discrimination, but not detection tasks, in contrast to our study. The researchers found a significant reduction in contrast sensitivity (CS) with both $+2.00 \mathrm{D}$ and $+4.00 \mathrm{D}$ ADDs in peripheral condition, but with the central CS test, a decrease in CS was found only with medium $(+2.00 \mathrm{D})$, but not with high (+4.00 D), ADD [19]. This finding agrees with our results showing that dVA was significantly reduced with the use of ADD2 but not ADD4.

We found a negative effect of peripheral additions on AR, regardless of the central distance zone size, which was reflected in the flattened slope of AR function with ADD4 (slope 0.74), as well as with ADD2 (slope 0.73) compared to ADD0 (slope 0.95). Interestingly, lenses with high additional power affected AR in the same way as those with lower addition, suggesting that high ADD does not influence accommodative functions to a higher degree than lenses with standard ADD power which are usually used for MC. Moreover, the accommodative lag was significantly higher with both additions used, as compared to single vision contact lenses, but only with the $4.5 \mathrm{~mm}$ and not with the $3 \mathrm{~mm}$ central zone size. The reason for that might be that high additions located close to the center of the visual field may be more easily "ignored" by the visual system than lower additions located at a greater distance from the center of the visual field. In MFSCLs designed 
for the presbyopic population, the increase of ADD is usually slower and the maximum ADD power is located at a greater distance in the periphery compared to tested polynomial progression zone design lenses. For example, Gong et al. found reduced AR with Biofinity MFSCL with $+2.5 \mathrm{D}$ of ADD, and the slope of the accommodative response function decreased from 0.82 for SVS contact lenses to 0.55 with MFSCL [22], which correspond to $33 \%$ of the function flattening. In our study, the ADD2 slope was 0.73 , which corresponds to $23 \%$ function flattening when compared to ADD0; however, interestingly, high ADD similarly flattened the slope of accommodative response function as medium $\mathrm{ADD}$ ( $22 \%$ when compared to ADD0). Kang et al. [23] investigated the influence of Proclear MFSCLs on accommodative functions using $+1.50 \mathrm{D}$ and $+3.00 \mathrm{D}$. They found that lower additions significantly increased accommodative lag when central and peripheral stimuli were used, but high additions affected accommodative functions only when peripheral stimuli were used, and no effect was found with central stimuli. This observation agrees with our findings that high additions located more centrally may affect accommodative functions to a lesser degree than lower peripheral additions, probably by causing the visual system to "ignore" the highly defocused peripheral retinal image.

Moreover, medium and high addition MFSCLs do not seem to influence AF, regardless of the ADD and central distance zone size. This finding is in agreement with results obtained by Gong et al. [22], who tested Biofinity Multifocal +2.50 ADD center-distance lenses. There are also other studies, e.g. by Chung et al. [24], which show that AF can be decreased in MFSCLS (both central distance and central near designs).

\section{CONCLUSIONS}

In almost all visual parameters measured in the current study, the lenses with high addition power (ADD4) influenced vision similarly to lower addition power (ADD2) lenses. The results for dVA were even better with higher than lower ADDs. We also showed that central zone diameter is a parameter that should be considered in fitting MFCLs for $\mathrm{MC}$, as it might influence PCS and AR. This might be easier to achieve in custom made designs. Conflicting findings from previous studies about visual performance in different designs of MFSCLs for MC require more studies using comparable protocols. Therefore, we can conclude that each design should be tested independently and recommendations for maximum addition power and central zone diameter should be given for clinicians using lenses in everyday practice.

\section{ACKNOWLEDGMENTS}

The authors would like to acknowledge Pascal Blaser from SwissLens SA for his advice and support with contact lenses from SwissLens SA, Prilly, Switzerland, and Joanna Paluch and Klaudia Wasilewska for technical help in the organization of the experimental group. The authors would like to thank Weronika Borek and Matthew Lyst for their advice on our manuscript and language corrections.

\section{DISCLOSURE}

This study was supported with products: contact lenses from SwissLens SA, Prilly, Switzerland.

The authors declare no conflict of interest.

\section{References}

1. Robboy MW, Hilmantel G, Tarver ME, et al. Assessment of clinical trials for devices intended to control myopia progression in children. Eye Contact Lens 2018; 44: 212-219.

2. Wildsoet CF, Chia A, Cho P, et al. IMI - Interventions Myopia Institute: interventions for controlling myopia onset and progression report. Invest Ophthalmol Vis Sci 2019; 60: M106-M131.

3. Sankaridurg P. Contact lenses to slow progression of myopia. Clin Exp 0ptom 2017; 100: 432-437.

4. Gifford KL, Richdale K, Kang P, et al. IMI - Clinical Management Guidelines Report. Invest Ophthalmol Vis Sci 2019; 60: M184-M203.

5. Wolffsohn JS, Calossi A, Cho P, et al. Global trends in myopia management attitudes and strategies in clinical practice. Cont Lens Anterior Eye 2016; 39: 106-116.

6. Efron N, Morgan PB, Woods CA, et al. International survey of contact lens fitting for myopia control in children. Cont Lens Anterior Eye 2020; 43: 4-8.

7. Li Q, Fang F. Advances and challenges of soft contact lens design for myopia control. Appl 0pt 2019; 58: 1639-1656.

8. Walline JJ. Myopia control: a review. Eye Contact Lens 2016; 42:3-8.

9. Flitcroft DI. The complex interactions of retinal, optical and environmental factors in myopia aetiology. Prog Retin Eye Res 2012; 31: 622-660.

10. Smith EL, 3rd, Campbell MC, Irving E. Does peripheral retinal input explain the promising myopia control effects of corneal reshaping therapy (CRT or ortho-K) \& multifocal soft contact lenses? Ophthalmic Physiol 0pt 2013; 33: 379-384.

11. Kang P, McAlinden C, Wildsoet CF. Effects of multifocal soft contact lenses used to slow myopia progression on quality of vision in young adults. Acta Ophthalmol 2017; 95: e43-e53.

12. Blaser P, Sexton I. Power profiles of peripheral additions using topographers with soft contact lenses in myopia control. In: Gobal Insight. 2019. https://www.contamac-globalinsight.com/articles/power-profiles-topographers-soft-contact-lenses-myopia-control/2019.

13. Montani G, Blaser P. Changes in relative peripheral refraction, HOAs and optical quality using a soft center distance multifocal contact lens with different additions and optical zones. International Myopia Conference, Birmingham 2017.

14. Schumacher JF, Thompson SK, OIman CA. Contrast response functions for single Gabor patches: ROI-based analysis over-represents low-contrast patches for GE BOLD. Front Syst Neurosci 2011; 5: 19.

15. Zhu Q, Liu Y, Tighe S, et al. Retardation of myopia progression by multifocal soft contact lenses. Int J Med Sci 2019; 16: 198-202.

16. Kollbaum PS, Jansen ME, Tan J, et al. Vision performance with a contact lens designed to slow myopia progression. Optom Vis Sci 2013; 90: 205-214. 
17. Schulle KL, Berntsen DA, Sinnott LT, et al. Visual acuity and over-refraction in myopic children fitted with soft multifocal contact lenses. Optom Vis Sci 2018; 95: 292-298.

18. Martins C, Amorim-De-Sousa A, Faria-Ribeiro M, et al. Visual performance and high-order aberrations with different contact lens prototypes with potential for myopia control. Curr Eye Res 2020; 45: 24-30.

19. Przekoracka K, Michalak K, Olszewski J, et al. Contrast sensitivity and visual acuity in subjects wearing multifocal contact lenses with high additions designed for myopia progression control. Cont Lens Anterior Eye 2020; 43: 33-39.

20. Zhao PF, Li SM, Lu J, et al. Effects of higher-order aberrations on contrast sensitivity in normal eyes of a large myopic population. Int J Ophthalmol 2017; 10: 1407-1411.

21. Fedtke C, Ehrmann K, Thomas V, et al. Association between multifocal soft contact lens decentration and visual performance. Clin Optom 2016; 8: 57-69.

22. Gong CR, Troilo D, Richdale K. Accommodation and phoria in children wearing multifocal contact lenses. Optom Vis Sci 2017; 94: 353-360.

23. Kang P, Wildsoet CF. Acute and short-term changes in visual function with multifocal soft contact lens wear in young adults. Cont Lens Anterior Eye 2016; 39: 133-140.

24. Chung J, Bakaraju R, Fedtke C, et al. Accommodative lag, facility and phoria with multifocal contact lenses. Invest Ophthalmol Vis Sci 2013; 54: 4251-4251. 